\title{
Interfacial debonding and damage progression in particle-reinforced composites
}

\author{
S. Womack \& M. S. Ingber \\ Department of Mechanical Engineering, University of New Mexico, \\ Albuquerque, NM, USA
}

\begin{abstract}
Decohesion between the matrix and inclusions in advanced multi-component composite materials is an important failure mechanism. In particular, the overall bulk strength of the composite depends strongly on interfacial damage and debonding. A 3-D model of interfacial decohesion is developed for a spherical particulatereinforced composite material. In this model, interfacial failure is initiated by the loss of ellipticity. A discrete constitutive equation is used to represent failure at the interface of the composite. Incremental displacement steps are applied to the model within the elastic range of the constituent materials and the interface is analyzed using the boundary element method to determine the progression of interfacial damage and debonding. The effective properties of the bulk composite are calculated at each incremental step.

Keywords:composite materials, decohesion model, incremental debonding, boundary element method.
\end{abstract}

\section{Introduction}

A composite is a manufactured material with two or more physically or chemically distinct phases. Particle composites typically contain a matrix material and inclusions. The interface between the inclusion and the matrix is a bonding surface, across which both weak and strong discontinuities occur [1]. Weak discontinuities are defined as discontinuous changes in the strain field across the limits of a narrow strain localization region over which the displacement field remains continuous. Under load, strong discontinuities may develop, causing the displacement field to become discontinuous and the strain field to become unbounded. 
Composites are designed to create desirable material properties such as increased strength, increased stiffness, decreased weight, corrosion resistance, and increased or decreased thermal and electrical conductivity. The overall performance of a composite depends on the material properties of each phase as well as the interfacial properties between the matrix and inclusions [2]. These material and interfacial properties govern how the material fails, including such failure modes as brittle fracture, ductile rupture, delamination, fiber pullout, yielding, and excessive deformation [3]. Composites commonly fail along the interface between the matrix and inclusion. This type of failure is called interfacial material failure and is defined as the formation of two new surfaces from a previously bonded interface between two materials. Interfacial decohesion is usually observed in composites with very low strength matrices relative to the inclusion, (i.e., ceramic inclusions in a pure aluminum matrix), while particle fracture usually occurs with a medium to high strength matrix $[4,5]$.

Given the significance of interfacial damage progression on the bulk strength and toughness of composite materials, it is not surprising that one of the major research areas in composites is the modeling of bonding interfaces between phases. The interfacial zone has been modeled in a number of ways, including as a narrow region of continuum with graded properties, as an infinitely thin surface with springs, and as a cohesive zone with traction-separation relations. Recently, the cohesive zone approach has become widely used. Needleman [6] was one of the first to apply the traction-separation cohesive relationship to the model. Needleman focused on a model that describes the evolution from initial debonding to decohesion and subsequent void growth. Needleman suggested a continuum approach where strain is defined not only in the continuous part of the body, but also at the discontinuous interface, and therefore, standard stress-strain constitutive equations can be considered everywhere. The major area of research using this technique is the determination of the traction-separation cohesive relationship, which has been modeled using various functions including linear [7], quadratic [8], polynomial [6], trapezoidal [9], bilinear [10], and exponential functions [11, 12].

Schreyer et al. [3] took a fundamentally diferent approach than Needleman, in which strain softening occurs after ellipticity is lost. In this approach, the onset of inelasticity or the loss of strong ellipticity are indicators of the progression of more complex material behaviors that lead to the progressive softening of the material [13]. The direct calculation of the strong discontinuity in displaceent can be determined because the discrete equation can be applied at the instant ellipticity is lost. Once decohesion is initiated on a surface of discontinuity, the adjacent continuum tends to unload into the elastic regime.

Han et al. [14] combined Schreyer's decohesion model with the boundary element method to develop a 2D simulation capability of interfacial decohesion of a fiber-reinforced composite material. The current research extends the work of Han et al. from 2D fiber-reinforced composites to 3D reinforced composites. In particular, boundary element analysis will be used in conjunction with the damage function proposed by Schreyer [3] at the interface between the matrix and inclusion to study interfacial damage of the composite. 


\section{Numerical formulation}

The computational domain considered in the current research is comprised of a matrix material with an embedded spherical inclusion. A boundary integral equation (BIE) is written for each of the two zoned-homogeneous, isotropic, elastic regions. Collocating the BIE at the boundary element nodes results in a system of linear equations relating the components of traction to the components of displacement. These equations can be represented symbolically as

$$
\left[H^{k}\right]\left\{\mathbf{u}^{k}\right\}=\left[G^{k}\right]\left\{\mathbf{t}^{k}\right\}
$$

where the superscript $k$ represents the region number, and $\left\{\mathbf{u}^{k}\right\}$ and $\left\{\mathbf{t}^{k}\right\}$ represent the components of displacement and traction, respectively, at the collocation nodes.

On the outer boundary of the matrix material, either the displacement or the traction is prescribed. On the interfacial nodes between the spherical inclusion and matrix, neither the displacement nor traction components are known a priori. The system of equations is closed by setting the following interfacial boundary conditions.

$$
\begin{aligned}
& \left\{u_{j}^{i}\right\}-\left\{u_{j}^{m}\right\}=[u] \\
& \left\{t_{j}^{i}\right\}=-\left\{t_{j}^{m}\right\}
\end{aligned}
$$

where $[u]$ represents a jump in displacement along the interface as discussed in the next section, the superscript $i$ represents the inclusion and superscript $m$ represent the matrix, and the subscript represents the global node within the appropriate region.

Initially, the interface between the matrix material and the inclusion is assumed to be perfectly bonded, that is, continuity of traction and displacement is assumed along the interface. In order to minimize the computation cost of the analysis, the displacement at which damage initiates is calculated and the decohesion analysis is started at that displacement. The displacement is then incrementally increased to study the progression of damage.

At each increment of displacement, the boundary integral equations are solved and the tractions along all interfaces are evaluated. The development of damage (decohesion) is determined through the use of a damage function $F$ given by

$$
\begin{aligned}
& F=\left[\left(\frac{\tau_{n}}{\tau_{n f}}\right)^{2}+\left(\frac{\tau_{t}}{\tau_{t f}}\right)^{2}\right]^{\frac{1}{2}}-f \quad \text { when } \quad \tau_{n} \geq 0 \\
& F=\left|\frac{\tau_{t}}{\tau_{t f}}\right|-f \quad \text { when } \quad \tau_{n}<0
\end{aligned}
$$

where $\tau_{n}$ is the normal component of traction, $\tau_{t}$ is the tangential component of traction, $\tau_{n f}$ is the value of failure initiation traction in a pure tensile mode, $\tau_{t f}$ is the value of failure initiation traction in a pure shear mode, and $f$ is the socalled softening function. The function $F$ is defined so that no damage occurs if 
$F<0$ and $F>0$ is not allowed. Damage develops only if $F=0$. The softening function $f$ is chosen so that $f=1$ for an undamaged interface and $f=0$ for a fully decohered interface. For $0<f<1$, a linear relationship is chosen between the absolute value of the jump in displacement $[\mathbf{u}]$ at the interface so $f$ is given by

$$
f=1-\frac{|[\mathbf{u}]|}{u_{0}}
$$

where the model parameter $u_{0}$ is the value of $|[\mathbf{u}]|$ at which complete decohesion has occurred. Although the choice of $f$ is somewhat arbitrary, in the special case of uniaxial tension, the softening function translates into a curve of normal traction versus normal displacement discontinuity in which case the area under the curve is the fracture energy, $G_{f}$.

After each increment of displacement, the damage function $F$ is evaluated at each interfacial node. If $F>0$, the threshold to initiate decohesion has been exceeded and is not allowed by the decohesion model. As an example, if the traction at an interfacial node had no tangential component, $F>0$ would imply that $\tau_{n f}$ had been exceeded. In order to drive $F$ to within a specified tolerance $\epsilon$ of 0 , the following algorithm is used. Nodes with the largest positive values of the damage function are chosen for softening, i.e., given a jump in displacement across the node between the inclusion and matrix.

The displacement discontinuities at the softened nodes are determined by the following evolution equation

$$
\Delta[\mathbf{u}]=\Delta \lambda \mathbf{g}
$$

where $[\mathbf{u}]=\left\{[\mathbf{u}]_{n}^{2}+[\mathbf{u}]_{t}^{2}\right\}^{\frac{1}{2}}, \mathbf{g}=\frac{\partial F}{\partial \tau}$, and $\Delta \lambda=\delta \lambda_{1}+\delta \lambda_{2}+\cdots$. The secant algorithm is applied to determine $\Delta \lambda$ in order to bring $F$ to within a tolerance $\epsilon$ of 0 . An initial small value for $\delta \lambda_{1}$ is assumed. This then provides the initial assumed value for $\Delta[\mathbf{u}]$ as shown in Eq. 6. This jump in displacement provides stress relief which is quantified by performing the boundary element analysis with the modified boundary conditions (Eq. 2). The damage function is then re-evaluated at the softened nodes. If $F$ is still positive and above the tolerance, $\delta \lambda_{2}$ is determined using the equation

$$
\delta \lambda_{2}=\delta \lambda_{1} \frac{F_{1}}{F_{0}-F_{1}}
$$

where the subscript on $F$ indicates the iteration number. The procedure is continued until $F<\epsilon$. During any iteration, if $|[\mathbf{u}]|$ reaches $u_{0}$, the boundary condition at that node is set to traction free indicating that the node is completely decohered.

\section{Damage and debonding propagation}

For all material tests, the outside dimensions of the material specimen is a cube with edge length 2 centered at the origin. The outside matrix material is aluminum with one spherical ceramic boron particle at a volume fraction of $10 \%$. The elastic 
material properties are given by $E_{a l}=67.51 \mathrm{GPa}, v_{a l}=0.35557, E_{B}=413.04$ $\mathrm{GPa}$, and $v_{B}=0.2$.

Two types of material tests are performed in this research, namely, a tensile test and a shear test. For the tensile test, an incremental uniform tensile displacement is applied to the top and bottom surfaces of the composite specimen. The effective Young's modulus for the composite undergoing the tensile test is calculated as

$$
E_{\text {eff }}=\frac{F_{n} L}{A \delta_{n}}
$$

where $F_{n}$ is the resultant normal force on the top or bottom surface of the specimen, $L$ is the specimen length, $A$ is the cross-sectional area, and $\delta_{n}$ is the applied normal displacement. The resultant force is calculated by integrating the tractions over the top and bottom faces.

For the shear test, an incremental uniform shear displacement is applied to the top and bottom surfaces of the composite specimen. The effective shear modulus is calculated as

$$
G_{e f f}=\frac{F_{s} L}{A \delta_{s}}
$$

where, in this case, $F_{S}$ is the resultant shear force on the top or bottom surface of the specimen and $\delta_{s}$ is the applied shear displacement.

The maximum interfacial traction is defined as

$$
\tau_{f}=\sqrt{\tau_{n f}^{2}+\tau_{t f}^{2}}
$$

where $\tau_{n f}$ and $\tau_{t f}$ are the normal and shear mode failure limits used to calculate the damage function $F$. For all cases, the maximum interfacial traction is chosen as $\tau_{f}=12 \mathrm{MPa}$. The parameter $u_{o}$, the maximum value that the displacement discontinuity reaches before debonding is complete, is chosen as $u_{o}=0.000046$, again for both the ductile and brittle failure models. The values of $\tau_{f}$ and $u_{o}$ are chosen to ensure that the interfacial bond will substantially fail before the matrix material yields.

Two cases are considered for both the tensile and shear test, namely, $\tau_{n f} / \tau_{t f}=$ 0.5 and $\tau_{n f} / \tau_{t f}=5.0$ to illustrate the difference between normal-force dominated failure and shear-force dominated failure.

\subsection{Tensile load results}

The first case considered is the single inclusion model undergoing a tensile load. Upon application of a normal displacement, the traction distribution on the top and bottom surfaces of the composite is non-uniform because of the inclusion. The magnitude of the traction vector normalized by the maximum interfacial traction, $\tau_{f}$, is plotted for three values of the applied strain in Fig. 1. As shown in Fig. 1(a) for the case of a composite consisting of an aluminum matrix with embedded ceramic spherical particle before the onset of interfacial damage, the traction distribution across the top surface increases towards the center of the surface 


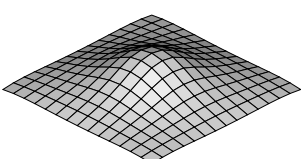

(a)

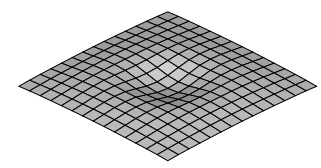

(b)

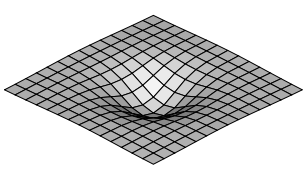

(c)

Figure 1: The magnitude of the traction vector along the top surface of the composite specimen undergoing a tensile test (a) before the initiation of interfacial damage, (b) after the initiation of interfacial damage but before the onset of debonding, and (c) after the onset of debonding.

where the gap between the surface and inclusion is smallest indicating the higher Young's modulus of the inclusion. The traction distribution shown in Fig. 1(b) for the case in which decohesion has been initiated but before any debonding has occurred is quite different. The damage along the interface at the north pole of the inclusion has reduced the load bearing capability resulting in a greatly reduced normal traction in that region. To compensate, the normal traction is increased in the adjacent nodes. Finally, as seen in Fig. 1(c) for the case in which debonding has occurred at the north pole, the normal traction in this region has been further reduced. Although the plots shown are for the normal-force dominated failure, the results for the shear-force dominated failure are qualitatively similar.

The normalized effective Young's modulus is defined by $E_{n}=E_{\text {eff }} / E_{\text {mat }}$ where $E_{\text {mat }}$ is the Young's modulus of the matrix material. The normalized Young's modulus is shown as a function of the applied normal strain in Fig. 2 for the shear-force dominated and normal-force dominated cases undergoing a tensile load. Initially,

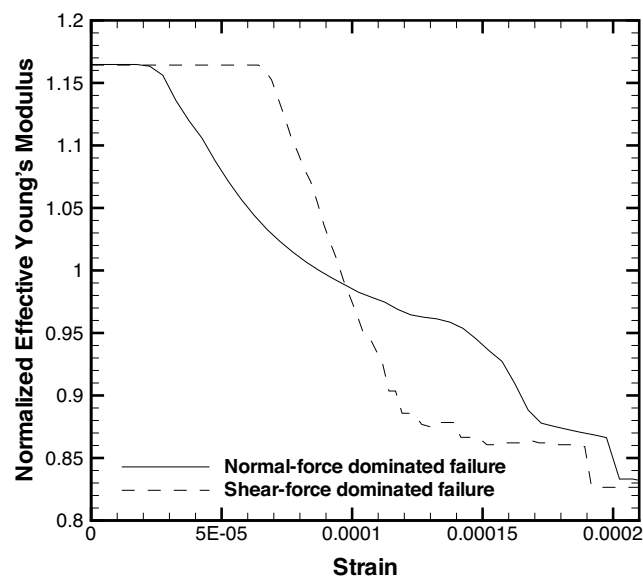

Figure 2: The normalized effective Young's modulus for shear- and normal-force dominated failure undergoing tensile loading. 
the normalized Young's modulus is given by $E_{n}=1.165$, indicating the enhanced stiffness of the composite caused by the spherical inclusion. As the incremental displacement is applied, the interface of the composite begins to experience damage when the bulk strain reaches $17 \mu \epsilon\left(\mu \epsilon=10^{-6} \mathrm{~mm} / \mathrm{mm}\right)$ and $64 \mu \epsilon$ for normal-force and shear-force dominated failure, respectively. Debonding of the first node occurs at approximately $100 \mu \epsilon$ regardless of the failure mode. The normalized Young's modulus plateaus after the stabilization of the equatorial region, at approximately $200 \mu \epsilon$ for the normal-force dominated failure and $160 \mu \epsilon$ for shear-force dominated failure.

There is a notable difference between the shear-force dominated and normalforce dominated failure modes. In the shear-dominated failure mode, damage initiates at either the north or south pole and then progresses from that pole towards the equatorial compressive zone before damage initiates at the opposite pole. The damage occurs only in one hemisphere from a strain of $64.0 \mu \epsilon$ until approximately $122 \mu \epsilon$. At that point, the effective Young's modulus remains relatively constant until a strain of approximately $142 \mu \epsilon$ as seen in Fig. 2. Beyond this strain, damage is initiated at the pole of the other hemisphere, and now damage and subsequent debonding progresses within the new hemisphere. For normalforce dominated failure, the progression of failure occurs simultaneously in both hemispheres.

\subsection{Shear load results}

The second case considered is the single inclusion model undergoing a shear test. When a shear displacement is applied to the top surface of the composite the traction distribution on this surface is again non-uniform. The magnitude of the traction vector normalized by the maximum interfacial traction is plotted for a case in which damage has been initiated in Fig. 3. For the shear test, damage initiates at a location $45^{\circ}$ from the vertical. The right-hand side of the top surface in the figure is the side closest to the damaged interface, and hence, the tractions are lower along the right side of the top surface compared to the left side because of the reduced load bearing capacity.

The normalized effective shear modulus is defined by $G_{n}=G_{\text {eff }} / G_{\text {mat }}$ where $G_{\text {mat }}$ is the shear modulus of the matrix material. The normalized shear modulus is shown as a function of the applied shear strain in Fig. 4 for the shear-force dominated and normal-force dominated cases undergoing the shear test. Initially, $G_{n} \approx 1.10$, indicating an increased shear modulus of the composite compared to the matrix material. As incremental shear displacement is applied, the interface of the composite begins to undergo softening when the bulk strain reaches $53 \mu \epsilon$ and $110 \mu \epsilon$ for normal- and shear-force dominated failure, respectively. Debonding of the first node occurs at approximately $228 \mu \epsilon$ and $200 \mu \epsilon$ for normal- and shear-force dominated failure. The normalized shear modulus plateaus after the stabilization of the rotated equatorial region, at approximately $268 \mu \epsilon$ for normaldominated failure and $210 \mu \epsilon$ for shear-dominated failure. As in the case of the tensile test, damage progresses in one hemisphere at a time for the shear-dominated 


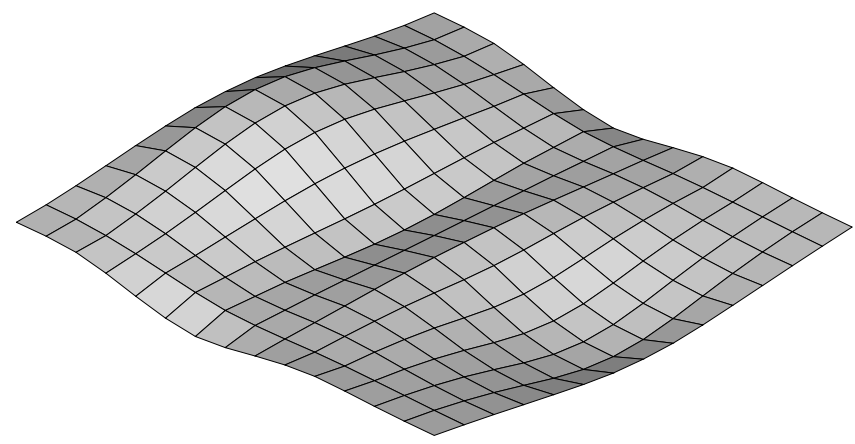

Figure 3: The magnitude of the traction vector along the top surface of the composite specimen undergoing a shear test after the onset of damage.

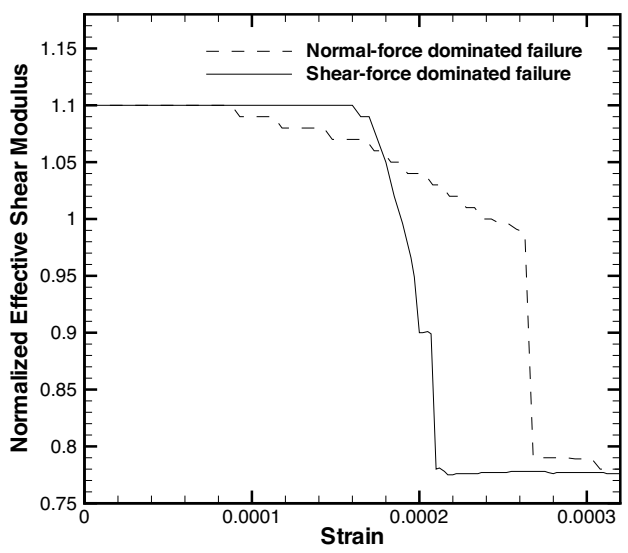

Figure 4: The normalized effective shear modulus for shear- and normal-force dominated failure undergoing shear loading.

failure case whereas damage progresses simultaneously in both hemispheres for the normal-dominate failure case.

\section{Conclusions}

A softening decohesion model has been combined with linear elasticity to describe the evolution of interfacial failure in spherical inclusion reinforced materials. As softening occurs, the sum of the work performed by the traction and the stored energy released by the material equals the energy dissipated. Progressive evolution of the debonding zones and the propagation of decohesion along the interface have been illustrated by incremental simulations. The material failure parameters, $\tau_{n f}$ and $\tau_{t f}$, play an important role in the decohesion process and may explain the difference between failure in ductile and brittle materials. In particular, if the ratio 
$\tau_{t f} / \tau_{n f}$ is small, then the material is more likely to fail in shear. In these cases once a portion of the interface becomes partially decohered, the damage zone slides tangentially along the interface until a compressive region is reached. On the other hand, if $\tau_{t f} / \tau_{n f}$ is large, then this shear mode failure is inhibited and damage tends to progress simultaneously on both sides of the inclusion. Decohesion has significant effect on the macroscopic response of the composite materials. In particular, the effective composite properties are adversely affected to the point where the partially decohered inclusion can actually diminish the effective shear and Young's moduli.

\section{References}

[1] K. K. Chawla, Composite Materials Science and Engineering, Springer Science+Business, New York, 1998.

[2] C. R. Dobert, R. Mahnken, E. Stein, Numerical simulation of interface debonding with a combined damage/friction constitutive model, Comp. Mech. 25 (2000) 456-467.

[3] H. L. Schreyer, D. L. Sulsky, S. J. Zhou, Modeling delamination as a strong discontinuity with the material point method, Comp. Meth. Appl. Mech. Engr. 191 (2002) 2483-2507.

[4] A. F. Whitehouse, T. W. Clyne, Cavity formation during tensile straining of particulate and short fiber metal-matrix composites, Acta Met. Mat. 41 (6) (1993) 1701-1711.

[5] L. Babout, E. Maire, R. Fougeres, Damage initiation in model metallic materials: X-ray tomography and modeling, Acta Mat. 52 (2004) 2475-2487.

[6] A. Needleman, A continuum model of void nucleation by inclusion debonding, J. Appl. Mech. 54 (1987) 525-531.

[7] G. T. Camacho, M. Ortiz, Computational modeling of impact damage in brittle materials, Int. J. Solids Struct. 33 (20-22) (1996) 2899-2938.

[8] V. Tvergaard, Effect of fiber debonding in a whisker-reinforced metal, Mat. Sci. Engr. 125 (2) (1990) 203-213.

[9] V. Tvergaard, J. W. Hutchinson, The relation between crack-growth resistance and fracture process parameters in elastic plastic solids, J. Mech. Phys. Solids 40 (6) (1992) 1377-1397.

[10] J. Segurado, J. LLorca, A new three-dimensional interface finite element to simulate facture in composites, Int. J. Solids Struct. 41 (2003) 2977-2993.

[11] A. Needleman, An Analysis of Decohesion Along an Imperfect Interface, Int. J. Fracture 42 (1) (1987) 21-40.

[12] A. Salvadori, A symmetric boundary integral formulation for cohesive interface problems, Comp. Mech. 32 (2003) 381-391.

[13] J. Oliver, Modeling strong discontinuities in solid mechanics via strain softening constitutive equations Part 1, Int. J. Num. Meth. Engr. 39 (1996) 35753600 .

[14] R. Han, M. S. Ingber, H. L. Schreyer, Progression of failure in fiberreinforced materials, Comp. Mats. Cont. 4 (3) (2006) 163-176. 